\title{
Design and Field Evaluation of REMPAD: A Recommender System Supporting Group Reminiscence Therapy
}

\author{
Yang Yang ${ }^{1, *}$, Niamh Caprani ${ }^{1}$, Adam Bermingham ${ }^{1}$, Julia O’Rourke ${ }^{2}$, Rónán Col- \\ lins $^{2}$, Cathal Gurrin ${ }^{1}$, and Alan F. Smeaton ${ }^{1}$ \\ ${ }^{1}$ INSIGHT: Data Analytics Research Centre, Dublin City University, Ireland \\ \{yangyang, adam.bermingham, cgurrin\}@computing.dcu.ie, \\ \{niamh.caprani, alan.smeaton\} @dcu.ie \\ ${ }^{2}$ Department of Speech \& Language Therapy, Adelaide \& Meath Hospital, Tallaght, Ireland \\ jjulia.orourke, ronan.collins 2 eamnch.ie
}

\begin{abstract}
This paper describes a semi-automated web-based system to facilitate digital reminiscence therapy for patients with mild-to-moderate dementia, enacted in a group setting. The system, REMPAD, uses proactive recommendation technology to profile participants and groups, and offers interactive multimedia content from the Internet to match these profiles. In this paper, we focus on the design of the system to deliver an innovative personalized group reminiscence experience. We take a user-centered design approach to discover and address the design challenges and considerations. A combination of methodologies is used throughout this research study, including exploratory interviews, prototype use case walkthroughs, and field evaluations. The results of the field evaluation indicate high user satisfaction when using the system, and strong tendency towards repeated use in future. These studies provide an insight into the current practices and challenges of group reminiscence therapy, and inform the design of a multimedia recommender system to support facilitators and group therapy participants.
\end{abstract}

Keywords: Reminiscence therapy; dementia; recommender systems; user interface.

\section{Introduction}

Reminiscence is the process of recalling personally experienced events from one's past. Reminiscence therapy (RT) is a popular psychosocial intervention used for people with dementia. Used either within a structured group or in a one-on-one session, it aims to stimulate senses in order to encourage memory recollection. Studies have shown that RT intervention can lead to positive outcomes in enhancement of selfesteem, increased life satisfaction, improved social interaction, as well as reduced depression [1]. Reminiscence and RT involve the deliberate use of prompts or cues, for example photographs, music, and smells, to promote the recall of pleasant memories and group RT is an example of direct therapeutic intervention for individuals with dementia [2]. Advances in information technology have made it possible to bring group RT into the digital age, introducing multimedia obtained from the internet. 
This paper outlines the design process for a novel computerized reminiscence system, REMPAD (Reminiscence Therapy Enhanced Material Profiling in Alzheimer's and other Dementias). The main purpose of the system is to address limitations in the way current group RT is conducted by automatically recommending content. The contribution of this paper is to investigate the current practices and challenges experienced by facilitators and use this to guide the design and evaluation of REMPAD.

\subsection{Public Content for Personal Meaning}

Reminiscence therapy and life review, a process of examining one's life, success and failures, have proven to be successful methods to improve the mood of older people, including those with dementia [1]. For this reason, many digital solutions have focused on personal content to support people with dementia. For example, Yashuda et al. [4] proposed a system to use personalized content with predefined themes; Sarne-Flaischmann et al. [5] concentrated on patients' life stories as reminiscent content; and Hallberg et al. [6] developed a reminiscence support system to use lifelog entities to assist a person with mild dementia.

Public or more generalised content are now being recognised as valuable reminiscence prompts, from which individuals obtain personal meaning. The benefit of this type of content is that different people have their own memories associated with a public event, which can stimulate conversation about shared experiences and interests, as well as personal reminiscence. André and colleagues [7] explored the concept of workplace reminiscence by creating personally evocative collections of content from publicly accessible media. Other studies examined the use of interactive systems, displaying generalized content to support people with dementia in clinical settings, such as hospitals or nursing homes. For example, Wallace et al. [8] designed an art piece for people with dementia and hospital staff to interact with. This consisted of a cabinet containing themed globes, which when placed in a holder initiated videos displayed on a TV screen, which were based on the associated theme, for example nature, holiday, or football. CIRCA, an interactive computer system designed to facilitate conversation between people with dementia and care staff, used a multimedia database of generic photographs, music and video clips to support reminiscence [9]. Astell et al. maintain that generic content is more beneficial than personal content as it promotes a failure-free activity for people with dementia, as there are no right or wrong memories in response to the stimuli.

However, what all these systems have in common is that their content is static and requires uploading and selection by either system developers or reminiscence facilitators. Multimedia websites potentially hold a wide range of subject matter that can be easily accessed. One question naturally arises: can we leverage the extensive range of online multimedia content, so that the reminiscence experience is maximized? We postulate that video sharing websites, such as YouTube ${ }^{1}$, are a valuable tool in promoting interaction and social engagement during group RT [10].

\footnotetext{
${ }^{1}$ YouTube is a video sharing website on which users can upload, view and share video content, including movie, TV and music video clips, as well as amateur content (www.youtube.com).
} 


\section{The REMPAD System}

REMPAD is a software system designed to facilitate group RT for people with Alzheimer's and other dementias. REMPAD offers a novel solution for group RT in that it uses intelligent classifiers to recommend publicly accessible videos from the Internet (e.g. YouTube) based on the group participants' profile, interests and hobbies [3]. The system also learns and adapts to group preferences with continued usage. This is obtained through a short feedback form which the facilitator fills out after each video clip and again at the end of the RT session. By automatically recommending video clips that are relevent to the group members experiences, the facilitors can focus on the group members' conversations, rather than the technology.
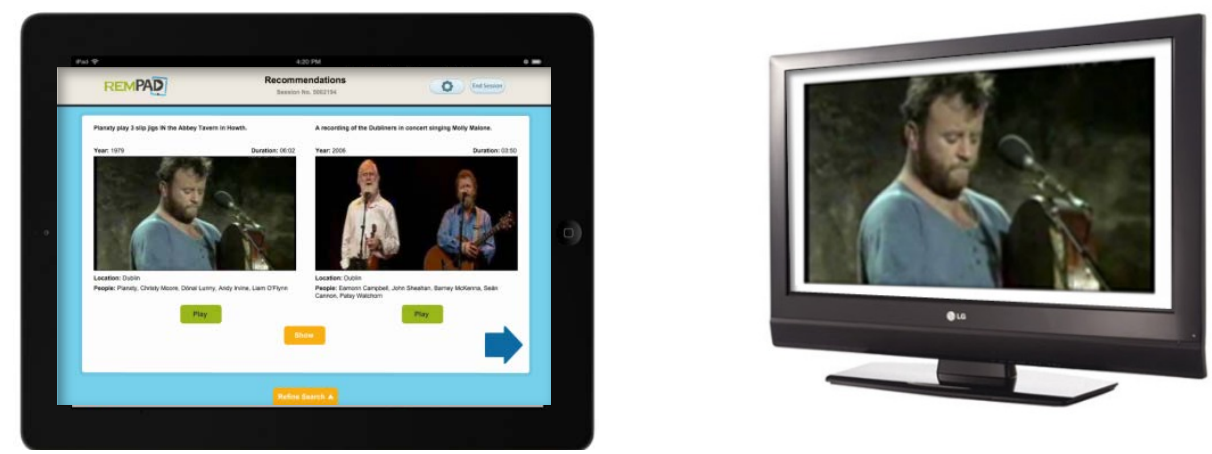

Fig. 1. The REMPAD system: facilitator view on tablet PC (left) and therapy participants view on large TV monitor (right).

The system has two hardware components: (1) a tablet computer, such as an iPad, which the RT facilitator interacts with, and (2) a TV monitor which displays video clips to the group members (see Fig. 1). Video clips are curated and annotated to ensure only high quality videos are displayed in the therapy session. This process is described in [3]. There are three main functions of REMPAD:

1. Participant profile - Personal information is recorded on a one off basis for each person who attends an RT session. This includes life history (age, locations of residence etc.) and their personal interests (music, fishing etc.) and is used to inform the system about which cues are likely to stimulate conversation.

2. Video recommendations - Once the RT facilitator logs onto the system they can begin an RT session. The aim is to provide cues to stimulate reminiscence and conversation. A binary choice of video clip is presented to the facilitator with video annotation. The facilitator can verbally relay this choice to the group. If one of these options act as a cue, the facilitor can select it to play on the TV screen. If the facilitator believes the group would benefit from seeing the choice, the facilitator can show a still image of both video clips side-by-side on the TV screen. If one of the options prompts reminiscence, the facilitator can select the video for the group to view. If neither are appropriate the facilitator can view 
the next two recommended videos. The videos are presented in a ranked order according to the group's aggregated profile.

3. Video feedback - While a video is being viewed by the group participants, the facilitator has the option to complete a short feedback form where they can rank on a 5-point scale the group satisfaction with the video, and also whether the video stimulated a positive, neutral or negative reaction for each participant. This information has potential value for both the facilitator's reports, and also to improve automatic recommendations.

Additional functions, such as providing end of session feedback, access to favourite and previously viewed video clips are also available. These were specifically requested by facilitators throughout the user-centred design (UCD).

\section{Design and Evaluation of REMPAD}

Healthcare systems are characterized by complex user requirements and information-intensive applications. Usability research has shown that a number of potential errors can be reduced by incorporating users' perspectives into the development life cycle [11]. Thus, employing a UCD approach throughout the development cycle, can lead to high quality intelligent healthcare systems. In order to conduct a UCD research study, we need to define user characteristics, tasks, and workflow to understand different stakeholder needs.

\subsection{Participant Sample}

The primary stakeholders of the REMPAD system are the facilitators who lead group RT sessions and interact directly with the system. For this study we focused on how the system supports these users to conduct the RT sessions. The participant sample consisted of 14 health professionals, including 7 speech and language therapists (SLTs) and 7 activity coordinators with nursing, counselling or social care qualifications. All participants currently run RT sessions in hospitals, day care centres or residential nursing homes. The 7 SLTs participated in Study 1 (interviews) and 2 (prototype testing), and the 7 activity coordinators participated in Study 3 (field evaluation). Throughout this paper we refer to the group RT leaders as facilitators.

The secondary stakeholders of the system are the therapy participants - people with dementia who attend the RT sessions. Although these participants do not directly interact with the tablet PC, information is displayed to the group through the TV monitor and information is also relayed through the facilitator. Current practice requires the facilitator to make subjective judgments after a session regarding the success of the material used in RT sessions to support inter-group interaction and their communication, mood and well-being. This was the method we used to gauge secondary stakeholder satisfaction in our field trials in Study 3. 


\subsection{User-Centred Design Process Overview}

The study was designed in 3 parts: (1) exploratory interview, (2) low-fidelity prototype test, and (3) field evaluation. We implemented findings from each stage into the system design which we then re-examined. We now discuss these methods.

\subsection{Study 1: Exploratory Interviews}

Interviews. The purpose of the exploratory interviews was to understand current RT practices, the types of technology used in these sessions if any, and the challenges that facilitators experience during these sessions. The interviews were semistructured in that a standard set of questions were prepared, however if the facilitator introduced a new topic, this was further explored. The types of questions that the facilitators were asked included: what types of technology do you use during a RT session? Do you prepare material before a group session? What are the challenges you experience? The interviews were audio recorded and later analysed by the research team for emerging trends. The findings are divided into four categories: current practices; technical skills; session challenges; and technical challenges.

Current Practices. The facilitators spoke to us about their current RT practices using physical and digital prompts. It was common for them to run RT in blocks e.g., one session per week over six weeks. Each facilitator may work with several groups, in several different locations. It was most common for them to use paper-based objects in these sessions, such as photos, newspaper clippings, and printed images. Physical objects were selected for their texture and smell to stimulate memories, for example polish or lavender. Music and video clips displayed on a TV screen or passed around via a laptop were also used. It was noted that shorter clips are preferable because these can hold the group's attention for longer.

The most common method used throughout the RT sessions was to begin with general or current themes. The reason for this was because the facilitator may not know participants' background or interests, and also to gently inform them of current issues, such as presidential elections etc. The conversation would then develop from these prepared topics. After the session, the facilitator would write up a report on what material or topics worked well to help prepare them for the following session.

Technical Skills. Through the interviews, we learned that the facilitators had different levels of technological expertise, from novice $(n=1)$, average $(n=5)$, to above average $(n=1)$ skills, and some $(43 \%)$ had little or no exposure using tablet PCs. There were varying levels of exposure to video sharing websites, ranging in use from twice a week for RT sessions to not at all. These characteristics pose a need for clear and intuitive interfaces with easy-to-use interaction modalities. Despite this, all participants embraced the idea of using a tablet device for promoting interaction, and using online videos as stimulus to help them conduct group RT sessions.

Technical Challenges. The facilitators reported experiencing several challenges when using technology in the RT sessions. For example, internet connectivity might 
be very good in some sections of a hospital but poor in others, and this needs to be taken into consideration when planning a session. Some locations also have blocked access to certain websites, including YouTube, and facilitators have to acquire permission to access it. This problem was resolved in the locations where access to Internet content was considered necessary for therapy sessions.

Another challenge that facilitators experienced was technology availability. The therapy participants require a large screen with high volume to accommodate those with vision and hearing difficulties. One of the participants said that when she accesses material on a computer it is necessary to pass the computer around the group so that each of them can see it. It was also reported that there is pressure to maintain the conversation with the group, ensuring all participants are included, while also trying to prepare material for the next topic.

Session Challenges. Facilitators told us that most of their working time is spent preparing for sessions, searching for appropriate material based on previous discussions or group preferences. On one hand, this meant that the facilitators were confident that the material would stimulate conversation, but it also meant that topics were fixed and did not allow for spontaneous deviation. Five of the seven facilitators had used video websites (such as YouTube) during their sessions to support spontaneous deviation. They reported difficulties finding content about a topic before the conversation drifts onto another topic. Currently, the practice is to prepare a number of video clips prior to the RT session to ensure that they are good visual and sound quality. These are used as a fall-back strategy if new videos are not successful. The facilitators also said that they prepare paper-based prompts should technical issues arise. However these prompts are static and require the facilitator to carry around a large bag of material with them. It was also mentioned that it would be preferable to use video clips or images that had positive results in the past, but these can be difficult to re-locate, particularly when the facilitator is under pressure to maintain conversation during a session.

The facilitators commented on the challenge of preparing for a group RT session when they do not know the participants' or group's preferences. This is most likely at the beginning of a block of sessions, as each week the facilitator will learn about the group interests. However, it was noted that there can be challenges learning about an individual's interests if they are unable to suggest topics or interact with the group, and it can be unhelpful to direct attention to them by putting them on the spot.

These findings highlight how facilitators would benefit from having easy access to participants' profile information, interests, and automatically recommended material that is inclusive for all (or as many as reasonably possible) group members.

\subsection{Study 2: Low-Fidelity Prototype Testing}

Use Case Walkthrough. The best way to present the technology behind the proposal is through a worked example. Based on the functional requirements provided, we created initial wireframe prototypes of the REMPAD system, consisting of a series of use cases. Wireframes were designed for facilitator interaction on the tablet computer 
and the TV display for therapy participants. In total, 12 use cases were created. Example use cases include: Start a new session; Edit an existing group; Browse video clips; and Enter feedback (see Fig. 2). A use case walkthrough was undertaken to familiarize participants (7 SLTs from Study 1) with the proposed task flow and interaction paradigm of the prototype system. Immediately after the walkthrough, there was a discussion with the facilitators to gather feedback on the system design.
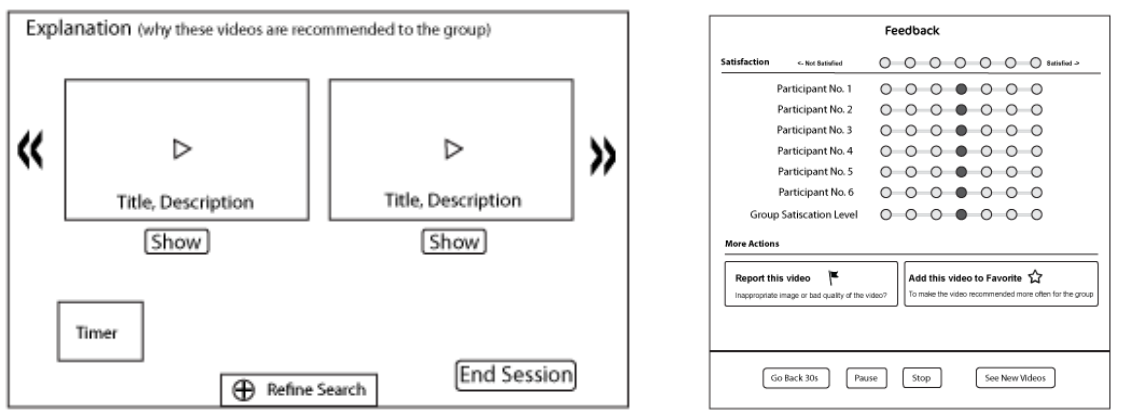

Fig. 2. Example wireframe screens used in use case walkthrough method.

Findings. Participants expressed high enthusiasm and positive response towards the initial prototype design. Overall, the facilitators thought the design was simple and straightforward, and that users with low technology experience would feel comfortable interacting with it. The main areas of discussion were focused on content recommendation and session feedback.

Content Recommendation. One of the crucial elements of an intelligent reminiscence system is to offer customizable content to users. Diversity exists inside a group in areas of individual backgrounds, interests and preferences. As one of the facilitators mentioned, "the biggest challenge is finding relevant videos". The facilitators were very enthusiastic about this feature and emphasised the importance of including the age of the person and place of birth into the recommender criteria. It was noted that automatic recommendation would save facilitators a significant amount of time, which is currently used planning RT sessions and would allow them to interact with the group rather than searching for appropriate material. Furthermore, as the system continues to learn about group preferences through facilitator feedback, new videos are continuously being recommended, enhancing the dynamic nature of the sessions.

The presentation of the videos was also discussed with the facilitators. It was decided that an option of two videos at a time was preferable as the facilitator could then relay this choice to the group without overloading them. Information about the video is also necessary so that the facilitator can have some knowledge about the subject being discussed. Finally, facilitators emphasised the importance of having control over topics. Maintaining the current practice of beginning a session with general topics and moving into more specific topics, facilitators said that they would use the recommended videos for the most part, but would like to have the option to search for a video based on how a conversation develops. Design alternatives were displayed to participants to search for a topic, or refine by category. We decided that the most appropriate design would be to include a search bar, which the user could refine ac- 
cording to a different year or decade. The ability to save successful video clips into a favourites section for future sessions was also requested by participants.

Session Feedback. Another challenge highlighted in building an intelligent reminiscence system is to ensure content is of high quality. In order to maximize group reminiscence experience, it was proposed that the recommendation engine should monitor patients' engagement levels, and adapt based on real-time user feedback. We designed the feedback screen layout as showed in Fig. 2. After each video, the group facilitator enters individual patient and group reactions to the presented video, so the selection of videos is improved in future sessions. However, we were unsure whether this function would add too much burden on the facilitator. During the discussion stage, participants unanimously confirmed that this level of feedback was achievable and understood and valued the benefit. The facilitators reported that they currently use pictures and icons to rate group satisfaction and topics discussed etc., in order to keep track of group progress. It was suggested that an end of session feedback report also be included in the system for the facilitator's records. This feedback was used to improve user interface design and justify design decisions, which were then implemented into a fully functional REMPAD system.

\subsection{Study 3: Usability Evaluation}

The REMPAD system was trialled over 54 RT sessions with 7 facilitators in 6 different locations over a period of several weeks. The facilitators used video clips recommended by the REMPAD system, sourced from YouTube, as cues to encourage reminiscence and conversation in the RT sessions. The purpose of the field trial was to investigate the use and usability of the system. We were also interested in investigating the performance of different configurations of our recommender algorithim, the results of which are discussed in [3]. The facilitators were given a post-trial usability and user experience questionnaire to complete which asked for feedback on the perceived usefulness of the system and system features, the ease of use for individual tasks, their satifaction with the system output and the overall usability of the system.

Findings. Overall, 362 recommended video clips were played during the RT sessions with an average of 6.7 per session. The facilitators reported the main benefit of REMPAD as being easy to use and requiring no preparation time. They also said that the therapy participants responded positively to the system, many being excited about seeing new technology. We asked facilitators to rate on a 5-point Likert scale the usefulness and usability of the system. The results of these can be seen in Table 1 . Overall the feedback was very positive. The facilitators found that the automatically recommended videos worked well for the therapy participants and the group as a whole. Only the search feature was rated as not very useful and it could be seen from our activity logs that this feature was rarely used.

The usability, learnability, efficiency to access information, response time, aesthetics and satisfaction using the system were all rated highly. The participants also reported the system tasks to be easy, such as setting up the system before a session, 
Table 1. Participants reports for the usefulness of REMPAD features (left) and the usability of the system (right), 5-point Likert scale from negative to positive (1-5).

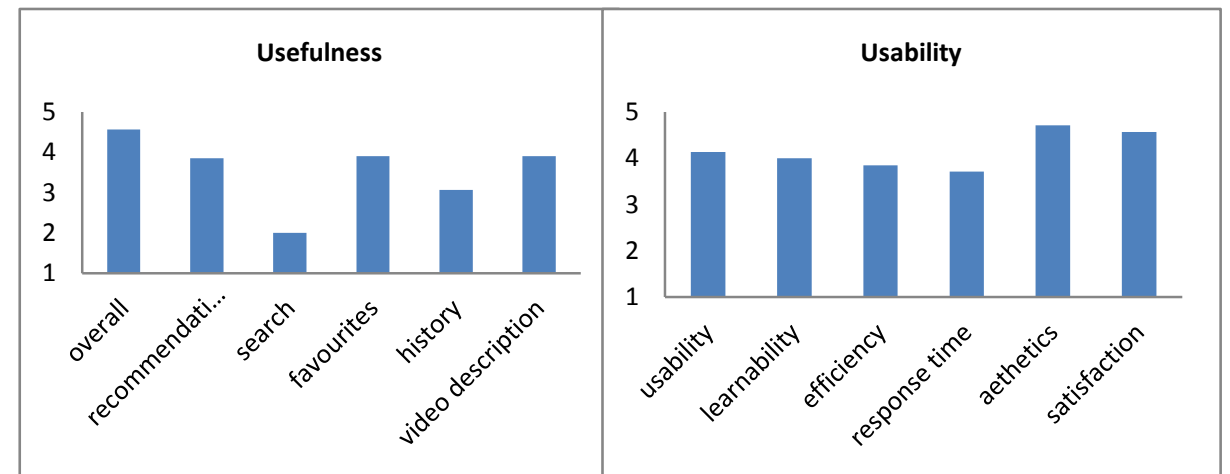

logging in, setting up participant profiles and groups, choosing relevant videos and entering video feedback. It was also found that the visual quality of videos was consistently good. However, intermittent problems did arise. For example, slow Internet connection in 3 of the locations meant that in some situations the videos took time to load and the therapy participants would lose interest. Problems with the sound being inconsistent were also reported. The facilitators suggested that a wider range of therapy participants other than older adults with dementia would benefit from REMPAD use, for example older and younger adults with acute brain injury.

In summary, the field evaluation provided us with a deeper understanding of how a multimedia recommender system is used in group RT sessions. The results from our trials are positive and indicate where future developments are needed. These findings will guide the next generation of REMPAD towards the deployment of the system into clinical settings.

\section{Conclusions}

In this paper we described the design and evaluation of a multimedia recommender system for group RT. We applied UCD techniques to address the challenges of current RT practices and explore potential solutions to these challenges. The problems that RT facilitators reported with their current methods included: spending a large portion of their time preparing material for the sessions; not knowing enough personal information about therapy participants to prepare relevant cues; and finding appropriate digital content whilst at the same time maintaining a conversation with the group. REMPAD supports facilitators by providing content that is relevant to an individual's interests and life history, as well as the shared interests for the group as a whole. Automatically recommending appropriate video content to cue group reminiscence could significantly reduce a facilitator's workload, allowing them to focus their time and attention on therapy participants rather than the technical equipment. Through our interviews, prototyping and field evaluations with RT facilitators and therapy participants, we have designed a novel, easy-to-use and, above all, useful system to facilitate group RT. 


\section{Acknowledgements}

This work is supported by Science Foundation Ireland under grant SFI/12/RC/2289 and Enterprise Ireland under grant CF/2011/1318.

\section{References}

1. Woods, B., Spector, AE., Jones, CA., Orrell, M., Davies, SP.: Reminiscence Therapy for Dementia. Cochrane Database Syst. Rev. 2 (2005)

2. Kim, E., et al.: Evidence-Based Practice Recommendations for Working with Individuals with Dementia: Group Reminiscence Therapy. J. Med. Speech Lang. Pathol. 14, 23-34 (2006)

3. Bermingham, A., O’Rourke, J., Gurrin, C., Collins, R., Irving, K., Smeaton, A.F.: Automatically Recommending Multimedia Content for use in Group Reminiscence Therapy. In: $1^{\text {st }}$ ACM MM Workshop MIIRH. ACM, Spain (2013)

4. Yasuda, K., Kuwabara, K., Kuwahara, N., Abe, S., Tetsutani, N.: Effectiveness of Personalized Reminiscence Photo Videos for Individuals with Dementia. Neuropsych Rehab. 19, 603-619 (2009)

5. Sarne-Flaischmann, V., Tractinsky, N.: Development and Evaluation of a Personalized Multimedia System for Reminiscence Therapy in Alzheimer's Patients. Int. J. Social and Humanistic Computing, 1, 81-96 (2008)

6. Hallberg, J., Kikhia, B., Bengtsson, J., Sävenstedt, S., Synnes, K.: Reminiscence Processes Using Life-Log Entities for Persons with Mild Dementia. In: $1^{\text {st }}$ International Workshop on Reminiscence Systems, pp.16-21. Cambridge, UK (2009)

7. André, P., Sellen, A., Schraefel, m.c., Wood, K.: Making public media personal: Nostalgia and Reminiscence in the Office. In: 25th BCS Conference on HumanComputer Interaction, pp. 351-360. Newcastle, UK (2011)

8. Wallace, J. Thieme, A., Wood, G., Schofield, G., Olivier, P.: Enabling Self, Intimacy and a Sense of Home in Dementia: An Enquiry into Design in a Hospital Setting. In: SIGCHI Conference on Human Factors in Computing Systems, pp. 2629-2638. ACM, Texas (2012)

9. Astell et al. (2009). Involving Older People with Dementia and their Carers in Designing Computer Based Support Systems: Some Methodological Consideration. Univ. Access Inform. Soc. 8, 49-58 (2009)

10. O'Rourke, J., Tobin, F., O'Callaghan, S., Sowman, R., Collins, D.R.: 'YouTube': A Useful Tool for Reminiscence Therapy in Dementia? Age and Ageing 40, 742-758 (2011)

11. Hesse, B.W., Shneiderman, B.: E-Health Research from the User's Perspective. American J. Prev. Med., 32, 97-103 (2007) 